\title{
Marangoni convection in a micropolar fluid with feedback control
}

\begin{abstract}
The effect of feedback control on the criterion for the onset of Marangoni convection in a horizontal micropolar fluid layer is studied theoretically. The upper free surface is assumed to be non-deformable and the lower boundary is taken to be rigid and isothermal with fixed temperature and span-vanishing boundaries. A linear stability analysis is used and the Galerkin method is employed to find the critical stability parameters numerically. It is found that the onset of instability can be delayed through the use of feedback control.
\end{abstract}

Keyword: Marangoni convection; Micropolar fluid; Feedback control 